\title{
Cooperation with Potential Leaders in Evolutionary Game Study of Networking Agents
}

\author{
Jianlei Zhang, Chunyan Zhang and Ming Cao \\ Faculty of Mathematics and Natural Sciences, \\ University of Groningen, The Netherlands \\ Email: jianlei.zhang; c.zhang; m.cao@rug.nl
}

\author{
Tianguang Chu \\ College of Engineering, Peking University \\ Beijing, China \\ Email: chutg@pku.edu.cn
}

\begin{abstract}
Increasingly influential leadership is significant to the cooperation and success of human societies. However, whether and how leaders emerge among evolutionary game players still remain less understood. Here, we study the evolution of potential leaders in the framework of evolutionary game theory, adopting the prisoner's dilemma and snowdrift game as metaphors of cooperation between unrelated individuals. We find that potential leaders can spontaneously emerge from homogeneous populations along with the evolution of cooperation, demonstrated by the result that a minority of agents spread their strategies more successfully than others and guide the population behavior, irrespective of the applied games. In addition, the phenomenon just described can be observed more notably in populations situated on scale free networks, and thus implies the relevance of heterogeneous networks for the possible emergence of leadership in the proposed system. Our results underscore the importance of the study of leadership in the population indulging in evolutionary games.
\end{abstract}

\section{INTRODUCTION}

Cooperation among self-serving individuals is an essential underpinning of modern human societies and wildlife coexistence alike [1]-[4]. The key question is why an individual would ever absorb a reproductive cost to perform acts that would benefit unrelated members of its social group [5]-[9]. Thus, understanding the emergence of altruistic behavior in the context of Darwinian evolution remains a challenge, met by scientists from many different fields, who often resort to evolutionary game theory as a common mathematical framework [10]-[12]. The most widely used models of game theory for the study of cooperative behavior, such as the Prisoner's Dilemma Game and the Snowdrift Game, provide scenarios of evolutionary dynamics where defectors dominate cooperators [13]-[16]. These models embody the social dilemma of cooperation in which individuals can benefit from mutual cooperation but they can do better by exploiting the cooperative action of others.

It can be easily observed that players are not perfectly identical within a population. And, heterogeneity among players has been explored as a beneficial condition for cooperation in several forms [17]-[19]. Notable examples include the heterogeneity of interactions. Over the past decades it has become clear that the metaphor of networks - ensembles of discrete nodes connected by links - offers a powerful conceptual framework for the description and analysis of many real world systems [20], [21]. Further, the shift from evolutionary games on regular grids to complex networks can be considered as a step towards more realistic conditions [22]-[25], and it has been confirmed that cooperation can survive and bloom in some heterogeneously-structured models [26]-[30]. Besides, various models have explored the individual differences in the personality traits such as reputation [31], [32], boldness [33], responsiveness [34], trustworthiness [35], or teaching [36].

In spite of ample progress that has been accumulated recently, there are situations of practical relevance that still remain less explored. For instance, evidence of leadership behavior has been found in a number of species, where individuals have different influences. In practice, some individuals ('leaders') often have strong influence on collective behavior, whereas others ('followers') have less ability and tend to conform to the preferences of the leaders. Several papers have shown that leadership by setting a good example (of being cooperative) has a positive influence on the behavior of others [37]-[40]. However, which players can be perceived as leaders is still an unsolved mystery. Is leadership designated or emerges spontaneously due to individuals possessing qualities or experiences in certain situations, or because they are of a personality type that is generally more inclined to lead?

In this regard, instead of assigning leader roles to group members as investigated in other studies [37], [38], we aim to investigate the (possible) spontaneous emergence of leadership from an initially homogeneous population indulging in evolutionary games on complex networks. Alternatively, all agents are in almost identical states, except for the randomly assigned strategies and locations on the nodes of the embedded network at the outset of games. Thus, each player is characterized by an initial strategy, indicating the player's unique label. Notably, strategy update may occur according to imitation rules over the course of evolution. In each round after the first, if a player succeeds in enforcing its unique label to others, its personality label gets imitated and sustained. In our study, the aggregate times of being imitated in the evolution process can be treated as a measure of individual's influence, i.e., leadership. That is to say, a leader is an individual whose strategy is highly imitated by others, even though it may perform strategy imitation in decision making.

The structure of the paper is organized as follows. The next section describes the basic framework in full detail. Section III is devoted to the presentation of main findings. Section IV provides some discussion and concludes.

\section{MOdEL DESCRIPTION}

Assuming structured interactions, we consider a total of $N=10^{4}$ agents occupying the nodes of a network. To 
preserve comparability of the host topologies, we employ the regular ring network and the Barabási Albert Scale-free network (BASF) [41] respectively, for the purpose of better comparison options. Agents that are connected are referred to as neighbors and together they may encounter competitive situations. They are faced with the decision of becoming a cooperator or defector in a competitive scenario. Consequently, each individual $x$ plays $k_{x}$ two-person games by following the same strategy in every game it is affiliated with, as prescribed by the interaction network.

Here, the Prisoner's Dilemma Game (PDG) and Snowdrift Game (SG) are used as the competitive paradigms. We label the payoff parameters in line with the conventions: a cooperating player receives the 'reward' $R$ in case of mutual cooperation and the 'sucker's payoff' $S$ in case of being defected; a defecting player receives the 'temptation to defect' $T$ when the other player cooperates and the 'punishment' $P$ in case of mutual defection. By definition, a PDG satisfies the payoff relationships $T>R>P>S$, and the SG is characterized by $T>R$ and $S>P$. For the remainder of the paper, we start by rescaling the games such that each depends on a single parameter. For the PDG, we make $T=b>1$, $R=1$, and $P=S=0$, where $b$ represents the advantage of defectors over cooperators, being typically constrained to the interval $1<b<2$. For the SG, we employ $T=\beta>1$, $R=\beta-\frac{1}{2}, S=\beta-1$, and $P=0$, such that the cost-to-benefit ratio of mutual cooperation can be written as $r=\frac{1}{2 \beta-1}$ with $0<r<1$.

Starting with a uniform distribution of cooperation (C) and defection (D), the synchronous update process proceeds as follows. Step (i): we adopt the PDG and SG to represent pairwise interactions among agents respectively. In each generation, all pairs of individuals $x$ and $y$, directly connected, engage in a single round of a given game, their respective payoffs being stored as $P_{x}$ and $P_{y}$. The payoff of a player at site $x$ is accumulated from the $k_{x}$ evolutionary games. Step (ii): each agent imitates the strategy (label) of a neighbor of better performances. Whenever a site $x$ is updated, a neighbor $y$ is drawn randomly among its $k_{x}$ neighbors; the chosen neighbor takes over site $x$ with the probability given by

$$
W_{s_{x} \rightarrow s_{y}}=\frac{1}{1+\exp \left[\left(P_{y}-P_{x}\right) / K\right]}
$$

where $K$ is the uncertainty related to the strategy adoption. $K=0$ and $K \rightarrow \infty$ denote the completely deterministic and completely random selection of $y$ 's strategy $s_{y}$ respectively. While for any finite positive value, $K$ incorporates the uncertainties in the strategy update, where the better one's strategy is definitely imitated, but there is a probability of selecting the worst ones. Herein, we consider the simple situation for individuals' selection probability and apply $K=0.1$ in this paper.

As mentioned above, each player on node $x$ randomly adopts a character $(C$ or $D)$ at the start of the game, which will be the unique character of the player and is preserved through the study. If player $x$ randomly adopts cooperation (or defection) initially, its single label is $x^{\prime} C$ (or $x^{\prime} D$ ). Thus, every time player $x$ succeeds in enforcing its strategy on $y$, its label gets imitated and sustained. Thus, in our definition, the individual label survives if it still exists at the final state, and even some players' labels may be adopted by plenty of agents. We will refer to this subset of agents as 'leaders', provided the condition is satisfied, where their labels (strategies) get largely imitated by others. If some individual labels are frequently copied by others within the population, these phenomena can be viewed as the possible emergence of leaders. Thus, we employ this model as a benchmark to study the possible emergence of leadership.

\section{SiMULATION AND RESULTS}

We track the evolution of strategies by iterating the model for a maximus of $10^{6}$ time steps. Here, a stationary state is one in which no further changes in cooperation level of the whole population are possible. The average cooperation levels are the ratios of cooperators in the system, regardless of their respective labels. Equilibrium frequencies of cooperators are averaged over $10^{3}$ time steps after a transient of sufficient time steps. Results are averages over 100 independent realizations of both the networks and the initial conditions.

Let us first investigate the cooperation level at the final state. The top panels in Fig. 1 show the simulation results carried out for both the PDG and SG on regular ring graphs for different average degree $k$. It is easier for cooperation to emerge in SG than PDG. Compared with the regular ring network, these phenomena are specifically notable in BASF networks. Notice also that numerical simulations with different average degree $k$ change the general behavior of the fraction of cooperation. It is clear that both the topology of the networks and the game models strongly affect the outcome of cooperation evolution.
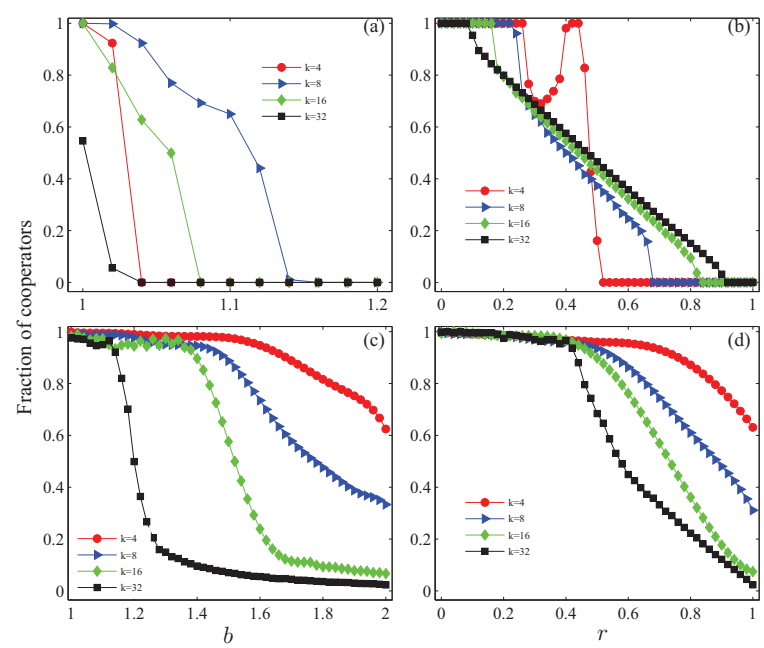

Fig. 1. (Color online) The resulting frequency of cooperators on different networks. Results shown as functions of the advantage of defectors $b$ for the PDG (left panels) and the cost-to-benefit ratio $r$ for the SG (right panels), for different average degrees. A notable enhancement in the cooperation level is observed in the BASF networks. Upper panels: Regular ring networks; Lower panels: BASF networks.

Then, we shift our attention to the fraction of surviving strategies of the whole population at the steady state, shown in Fig. 2, which reveals differences in network configurations. As mentioned, a surviving strategy is such a strategy that is adopted by at least one individual when the system converges to a stable state after a long enough evolution. As Fig. 2 
illustrates, the result is closely related to the variations of the topological structure and game types. For the PDG on regular ring network, we find that almost half of the population's labels disappear finally. Moreover, as $k$ grows the amount of the survival labels displays large increment, especially for low values of $b$ in the PDG on regular ring network. These phenomena are possible consequences of the increasing pairwise interactions on regular ring network, which dominates several microscopic differences of graphs and leads to similar well-mixed scenario that will decrease the spread possibility of individual labels.

However, alternative assumption on the population structures yields a significant difference on BASF networks. A remarkable decrease of the surviving strategies is found for large degree $k$. We interpret this finding by the fact that large average degree may lead to strongly heterogeneous interactions network, which is tightly associated with flushing cooperative states. Thus, the strategy update rule helps a small minority effectively guide a large group of followers. Another message is that the amount of surviving labels achieved on BASF networks is remarkably lower than those observed on regular ring network. As evidenced by results presented in Fig. 2(c), the fraction of surviving labels is below 0.1 over the entire range of the parameter $b$ for the PDG. This indicates that some individuals succeed in disseminating their labels among the population. This striking result points out that the ability of BASF to outperform the promotion of leaders on regular ring network is intrinsically due to the entanglement of interaction heterogeneities.

A further test on the SG is also included in Fig. 2 for comparison. Qualitatively, the systems show different behavior with PDG. For the SG on regular ring network, the number of surviving labels decreases with the game parameter $r$ raises. Gradually tracing $r$ downwards also brings the system to a state consisting of some leaders. According to Fig. 2(b), surviving strategies are clearly in the minority, especially at intermediate ranges of cost-to-benefit ratio $r$. Before moving forward, we briefly comment that, this crucial observation clearly indicates that potential leaders may emerge from spatially homogeneous population. Changing assumptions on the structured population from homogeneity to heterogeneity leads to lower values of the surviving labels. It has been argued that the key topological feature of a BASF network that promotes cooperation is the high connectedness of the hubs. Starting from a random distribution of strategies, hubs can win over the neighbors because their hub-specific high connectivity results in large cumulative payoffs. Therefore players that are linked to a hub will imitate its strategy, which eventually results in a cloud of homogeneous strategists around each hub. Individuals occupied large-degree nodes will pass their labels to others more easily. In the long run, such an evolutionary process strengthens the cooperative hubs and promotes the emergence of leaders. Moreover, higher average degree $k$ facilitates the emergence of possible leaders in such situation.

From fig. 2, we can also find that for harsh cooperative environments (high $b$ or $r$ ), leaders are more visible especially in BASF networks. For small values of $b$ or $r$, it is easier to spread their strategies for the cooperative hubs. Therefore, more cooperators survive in this situation. As the values of $b$ and $r$ grow the fraction of surviving labels displays large decrement. The reason may be that most leaders are cooperators because of the effects of cooperator clusters. We know that on graphs, clustering of cooperators help them maintain and spread their strategies. So, in harsh cooperative environments, fewer cooperator clusters can survive. Therefore, larger $b$ and $r$ make leaders more visible.
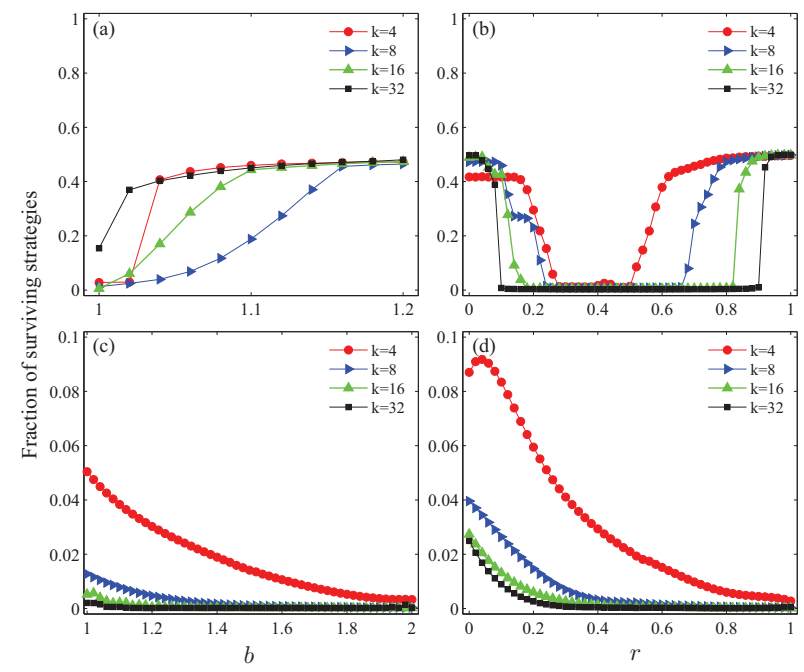

Fig. 2. (Color online) The fraction of surviving strategies amongst all players for different values of the average degree $k$, as functions of the advantage of defectors $b$ for the PDG (left panels) and the cost-to-benefit ratio $r$ for the SG (right panels). It can be observed that a minority of individuals succeed in disseminating their labels among the population. Upper panels: Regular ring networks; Lower panels: BASF networks.

As a further point, we verify the above numerical simulation results by means of mathematical analysis. In the BASF network, the probability that a vertex has $k$ edges follows a distribution of

$$
P(k)=\frac{2 m(m+1)}{k(k+1)(k+2)} \simeq 2 m^{2} k^{-3}
$$

where $m$ denotes that a new vertex will link $m$ different vertices that are already present in the system. In our proposed system, if individuals with degree $k>k_{0}$ survive at the final stationary state, we get the average degree of these surviving nodes by

$$
\bar{k}=\frac{\int_{k_{0}}^{N} 2 N m^{2} k^{-3} k d k}{\int_{k_{0}}^{N} 2 N m^{2} k^{-3} d k} \simeq 2 k_{0}
$$

Then, we calculate the fraction of surviving individuals as follows

$$
\int_{\frac{1}{2} \bar{k}}^{N} 2 m^{2} k^{-3} d k \simeq \frac{8 m^{2}}{\bar{k}}
$$

with a premise that individuals with more than $k$ edges can survive finally.

According to this, the theoretic analysis results and difference between theoretical and simulation are provided in Fig. 3. As evidenced by presented results, the difference between theoretical prediction and simulation results shows a gradually decreasing trend as the increasing game parameter $b$ or $r$. For low $b$ or $r$, the fraction of surviving individuals obtained by theoretical analysis is larger than that gained by 

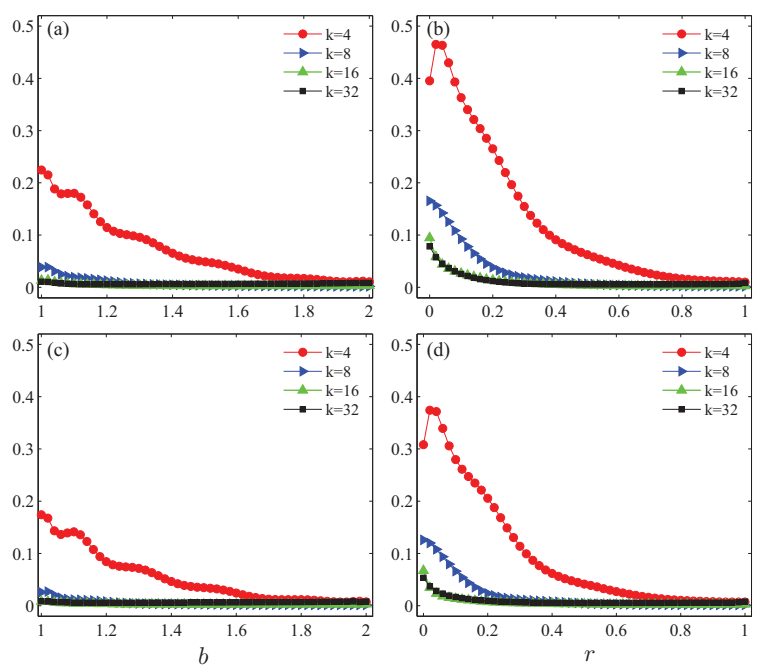

Fig. 3. (Color online) Upper panels: theoretical analysis results of the fraction of surviving strategies in the final stationary state whose degrees are more than $k$ on BASF networks; Lower panels: the difference between theoretical analysis and simulation results. Left panels: PDG; Right panels: SG. It can be observed that individuals with higher degrees indeed pass their strategies more easily than lower ones.

the numerical simulation. The reason lies in the fact that the theoretical analysis is based on the premise that all nodes with more than $k$ edges can survive finally. However, the non-zero values in Fig. 3(c,d) indicate that not all individuals with more than $k$ degrees could successfully pass their labels to the final steady state. The above mentioned phenomenon is primarily notable at low average degrees, e.g., $k=4$ in Fig. 3. However, in harsh cooperation environment (i.e. high $b$ or $r$ ) and BASF networks with larger average degrees, more individuals with large degrees can successfully pass their labels to the final stationary state. Thus, the theoretical analysis verify our conclusion that higher-degree players are more successful in influencing others.

To get a deeper understanding of the presented results, we further investigate the global intensity of the surviving cooperative labels. Fig. 4 offers such view. As can be seen from the figure, compared with results in Fig. 2, all the realizations lead the system to a configuration in which most strategists are cooperators among a wide range of $b$ or $r$ on BASF networks. For the PDG on regular ring network, the fraction of surviving cooperators appears lower than that on BASF network. In particular, larger average degrees on regular ring lead to a gradual but unavoidable shrinkage of the cooperator clusters and the according cooperation level. For the SG on regular ring network, a majority of players act as cooperators and can withstand being invaded by defectors at low $r$ values. This, however, is very short lived as defectors gradually gain the eventual dominance at high $r$ values.

Further, it remains of interest to examine the degrees of surviving labels emerging within the two employed games. When the interaction structure is characterized by a homogenous regular ring topology, each site is connected to its $k$ nearest neighbors. Evidently, the connectivity of the final surviving labels still stays at $k$ on regular ring networks, with the same number of neighbors. Now we only discuss
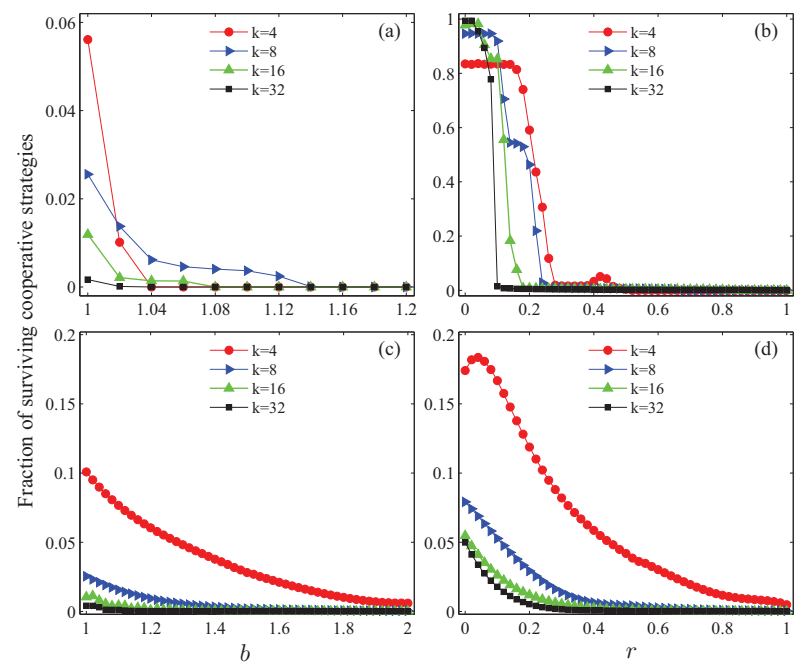

Fig. 4. (Color online) The fraction of surviving cooperative strategies amongst all players for different values of the average degree $k$, as functions of the advantage of defectors $b$ for the PD (left panels) and the cost-to-benefit ratio $r$ for the SG (right panels). The plots show that there is a relevant dependence on the underlying network. Upper panels: Regular ring networks; Lower panels: BASF networks.
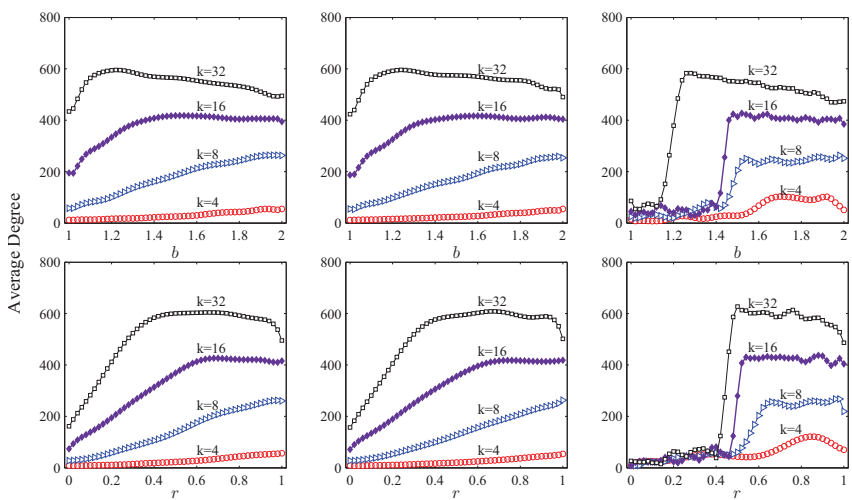

Fig. 5. (Color online) The average degree of the surviving strategists on the BASF networks. Panels depict for the PDG (upper panels) and the SG (lower panels). The plots reveal that surviving strategists occupy large-degree nodes. Left panels: Surviving strategies; Middle panels: Surviving cooperative strategies; Right panels: Surviving defective strategies.

the mentioned heterogeneous connectivity structures exhibiting scale-free properties, allowing us to capture relevant details of degree distributions of surviving labels. Fig. 5 shows a variation of degree distribution, when we tune the value of the mean degree $k$ of BASF network for the two games. These data refer to the final population degree (left panels), the cooperators (middle panels) and the defectors (right panels) respectively.

As indicated in Fig. 5, the remaining labels are those sites with large degrees, irrespective of which game applies. Also, we find that games on networks towards larger average degrees substantially enhance the emergence of large-degree leaders. Moreover, for the whole population, the present phenomenon affected by $b$ or $r$ is similar to coherence resonance, but that the optimal values for the increment of degrees differ in the two games. In addition, it can be observed that the overall impact of increasing $b$ or $r$ is exactly different on the two 
roles of cooperators and defectors. The results for cooperators show an increasing trend when the value of temptations to defect gradually increases. For defectors, its curves, obtained for the same parametrization, show a slowly decreasing trend when the value of temptations to defect is gradually increased. The reason is that with the increasing of $b$ or $r$, it is easier to survive for defectors but more difficult for cooperators.
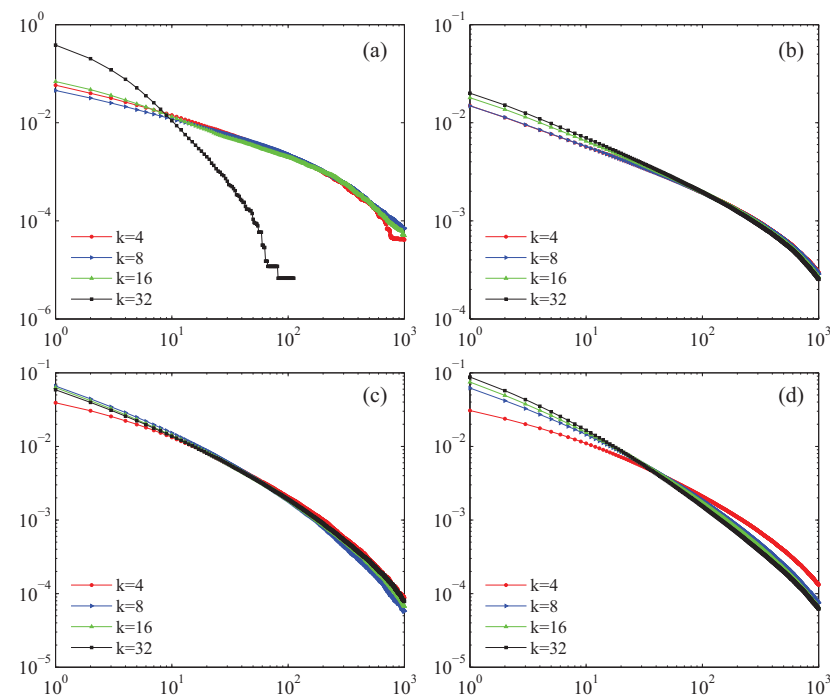

Fig. 6. (Color online) How individuals influence and be influenced by others in the strategy update process. Panels depict for the PDG (a,c) and SG (b,d). The $X$ axis is the times of an individual's strategy being imitated by others. The $Y$ axis is the ratio of times of an individual learning others accounting for the whole strategy transfer times. Results illustrate the heterogeneity among individuals' behavior. Upper panels: regular ring networks; Lower panels: BASF networks. The rest parameters are (a) $b=1.4$, (b) $r=0.2$, (c) $b=1.6$ and (d) $r=0.8$.

Finally, in order to reveal the main mechanism behind the remarkable presence of leadership, we investigate more specifically how individuals influence and be influenced by others in the strategy update process. We endow each player $i$ with an array $\left(x_{i}, y_{i}\right)$, where $x_{i}$ denotes the times player $i$ enforces its strategy on others in the whole evolution process, and $y_{i}$ denotes the times player $i$ learns others' strategies. It is noteworthy that the more an individual inclined to be a leader the larger value of $x_{i}-y_{i}$ is. Initially any player $i$ of the population has $x_{i}=1, y_{i}=1$. Here, each time player $i$ successfully passes its strategy on another player $j$, thus $x_{i}+1$ and $y_{j}+1$. Notice the fact that player $i$ is able to enforce its strategy on player $j$ implies that the former is more successful. In Fig. 6 we plot the ratio of the times of an individual $i$ learning others accounting for the whole strategy transfer times through evolution process, as a function of $x_{i}$.

Fig. 6 compares two series of numerical results obtained by varying the average degree $k$ on regular ring network (upper panels) and BASF (lower panels) consisting of $10^{4}$ sites respectively. For both games the value of the ratio $q_{i}$ decreases steadily as $x_{i}$ increases, albeit that the rate of the decrease slightly differs depending on the adopted games. This holds irrespective of the underlying structures. Notably, results indicate that the majority do not show strong leadership, and most of the strategy imitations are implemented by them.
However, as noticed by the examination of decreasing tendency with larger $x_{i}$, the results also correspond to a scenario where surviving strategies are less affected by others. Put another way, a small amount of individuals indeed possesses strong ability in affecting others. Here, the most intriguing still concerns the observed differentiation of individual ability in influencing others on regular ring network. Starting from a homogeneous situation, it is clear that the differentiation among individuals emerges in the evolution process. More importantly, it is irrelevant to the heterogeneity of individual connectivity. Thus, these results strengthen our above finding that leadership can potentially emerge from a homogenous population.

\section{CONCLUSions}

In a nutshell, by endowing each individual with a label (denoted by initial strategy) and tracing the headstream of each surviving label when the system converges to a stable state, we find that a few players indeed exert influence on the collective behavior and guide some followers. With the aid of extensive numerical simulations (performed on different networks and games), our intriguing results show that some individuals could gain leading roles despite the homogeneity of connectivity, especially for suitable average degrees where cooperators survive. Further, results also indicate that leadership, along with cooperation, may be substantially promoted by the usage of heterogenous neighborhoods. The influence of those individuals occupying hubs is significant that it may dictate the outcome of evolutionary dynamics. If cooperative hub-players can turn their 'followers' into cooperators, then they can have the stable and leading roles due to their superior payoffs. All the above phenomena indicate that there are possibly many ways to influence and enhance leadership among homogeneous members. Further investigations would be required to clarify the distinguished role of leaders, appearance of different time scales, and the interplay between leadership and cooperation.

\section{ACKNOWLEDGMENT}

This work was supported by the National Basic Research Program of China (973 Program, No. 2012CB821200) and the National Natural Science Foundation of China (No. 61273111). Chunyan Zhang, Jianlei Zhang and Ming Cao also acknowledge the support from the Erasmus Mundus project and the Dutch Organization for Scientific Research (NWO) (grant No. 82301006), the European Research Council (ERCStG-307207) and the EU INTERREG pro- gram under the auspices of the SMARTBOT project (SMARTBOT2012).

\section{REFERENCES}

[1] E. Fehr and U. Fischbacher, "The nature of human altruism," Nature, vol. 425, pp. 785-791, 2003.

[2] A. S. Griffin, S. A. West, and A. Buckling, "Cooperation and competition in pathogenic bacteria," Nature, vol. 430, pp. 1024-1027, 2004.

[3] W. D. Hamilton, "Geometry for the selfish herd," J. Theor. Biol., vol. 31, pp. 295-311, 1971.

[4] H. Brandt, C. Hauert, and K. Sigmund, "Punishment and reputation in spatial public goods games," Proc. R. Soc. Lond. B, vol. 270, pp. 1099-1104, 2003.

[5] E. Fehr and B. Rockenbach, "Detrimental effects of sanctions on human altruism," Nature, vol. 422, pp. 137-140, 2003. 
[6] F. Fiegna, Y.-T. N. Yu, S. V. Kadam, and G. J. Velicer, "Evolution of an obligate social cheater to a superior cooperator," Nature, vol. 441, pp. 310-314, 2006.

[7] K. R. Foster, G. Shaulsky, J. E. Strassmann, D. C. Queller, and C. R. L. Thompson, "Pleiotropy as a mechanism to stabilize cooperation," $\mathrm{Na}$ ture, vol. 431, pp. 693-696, 2004.

[8] M. Milinski, “A revolution in evolution,” Nature, vol. 471, pp. 294-295, 2011.

[9] M. A. Nowak, Evolutionary Dynamics. Cambridge, MA: Harvard University Press, 2006.

[10] H. Gintis, Game Theory Evolving. Princeton: Princeton University Press, 2000.

[11] J. Maynard Smith, Evolution and the Theory of Games. Cambridge, UK: Cambridge University Press, 1982.

[12] R. Axelrod, The Evolution of Cooperation. New York: Basic Books, 1984.

[13] M. Doebeli and C. Hauert, "Models of cooperation based on prisoner's dilemma and snowdrift game," Ecol. Lett., vol. 8, pp. 748-766, 2005.

[14] C. P. Roca, J. A. Cuesta, and A. Sánchez, "Evolutionary game theory: Temporal and spatial effects beyond replicator dynamics," Phys. Life Rev., vol. 6, pp. 208-249, 2009.

[15] J. Gore, H. Youk, and A. van Qudenaarden, "Snowdrift game dynamics and facultative cheating in yeast," Nature, vol. 459, pp. 253-256, 2009.

[16] J. Zhang, X. Chen, C. Zhang, L. Wang, and T. Chu, "Elimination mechanism promotes cooperation in coevolutionary prisoner's dilemma games," Physica A, vol. 389, pp. 4081-4086, 2010.

[17] E. Lieberman, C. Hauert, and M. A. Nowak, "Evolutionary dynamics on graphs," Nature, vol. 433, pp. 312-316, 2005.

[18] J. M. McNamara, Z. Barta, and A. I. Houston, "Variation in behaviour promotes cooperation in the prisoner's dilemma game," Nature, vol. 428, pp. 745-748, 2004.

[19] M. Perc and A. Szolnoki, "Coevolutionary games - a mini review," BioSystems, vol. 99, pp. 109-125, 2010.

[20] J. H. Fowler and N. A. Christakis, "Cooperative behavior cascades in human social networks," Proc. Natl. Acad. Sci. U.S.A., vol. 107, pp. 5334-5338, 2010

[21] M. A. Nowak and R. M. May, "Evolutionary games and spatial chaos," Nature, vol. 359, pp. 826-829, 1992.

[22] S. Boccaletti, V. Latora, Y. Moreno, M. Chavez, and D. Hwang, "Complex networks: Structure and dynamics," Phys. Rep., vol. 424, pp. 175-308, 2006.

[23] O. Gräser, C. Xu, and P. M. Hui, "Disconnected-connected network transitions and phase separation driven by co-evolving dynamics," $E P L$, vol. 87, p. 38003, 2009.

[24] M. Perc, "Chaos promotes ccoperation in the spatial prisoner's dilemma game,” EPL, vol. 75, pp. 841-846, 2006.

[25] T. Gross and B. Blasius, "Adaptive coevolutionary networks: a review," J. R. Soc. Interface, vol. 5, pp. 259-271, 2008.

[26] J. M. Pacheco, A. Traulsen, and M. A. Nowak, "Coevolution of strategy and structure in complex networks with dynamical linking," Phys. Rev. Lett., vol. 97, p. 258103, 2006.

[27] J. Zhang, C. Zhang, and T. Chu, "Cooperation enhanced by the survival of the fittest' rule in prisoner's dilemma games on complex networks," J. Theor. Biol., vol. 267, pp. 41-47, 2010.

[28] G. Szabó and G. Fáth, "Evolutionary games on graphs," Phys. Rep., vol. 446, pp. 97-216, 2007.

[29] R. Suzuki, M. Kato, and T. Arita, "Cyclic coevolution of cooperative behaviors and network structures," Phys. Rev. E, vol. 77, p. 021911, 2008 .

[30] M. Perc, "Evolution of cooperation on scale-free networks subject to error and attack," New J. Phys., vol. 11, p. 033027, 2009.

[31] A. Szolnoki and M. Perc, "Coevolution of teaching activity promotes cooperation," New J. Phys., vol. 10, p. 043036, 2008.

[32] — , "Emergence of multilevel selection in the prisoner's dilemma game on coevolving random networks," New J. Phys., vol. 11, p. 093033, 2009 .
[33] M. Wolf, G. S. van Doorn, and F. Weissing, "Life-history trade-offs favor the evolution of animal personalities," Nature, vol. 447, pp. 581585, 2007.

[34] M. Wolf, G. Van Doorn, and F. Weissing, "Evolutionary emergence of responsive and unresponsive personalities," Proc. Natl. Acad. Sci. U.S.A., vol. 105, no. 41, p. 15825, 2008.

[35] J. M. McNamara, P. A. Stephens, S. R. X. Dall, and A. I. Houston, "Evolution of trust and trustworthiness: social awareness favours personality differences," Proc. R. Soc. B, vol. 276, pp. 605-613, 2009.

[36] A. Szolnoki and G. Szabó, "Cooperation enhanced by inhomogeneous activity of teaching for evolutionary prisoner's dilemma games," $E P L$, vol. 77, p. 30004, 2007.

[37] W. Buckley, T. Burns, and L. Meeker, "Structural resolutions of collective action problems," Behavioral Science, vol. 19, no. 5, pp. 277-297, 1974.

[38] R. Johnstone and A. Manica, "Evolution of personality differences in leadership," Proc. Natl. Acad. Sci. U.S.A., vol. 108, pp. 8373-8378, 2011.

[39] P. L. Hooper, H. S. Kaplan, and J. L. Boone, "A theory of leadership in human cooperative groups," J. Theor. Biol., vol. 265, pp. 633-646, 2010.

[40] M. Van Vugt, S. F. Jepson, C. M. Hart, and C. De Cremer, "Autocratic leadership in social dilemmas: A threat to group stability," J. Exper. Soc. Psych., vol. 40, pp. 1-13, 2004.

[41] A.-L. Barabási and R. Albert, "Emergence of scaling in random networks," Science, vol. 286, pp. 509-512, 1999. 